\title{
How the COVID-19 Pandemic Affected the Accessibility and Quality of Health Services in Poland
}

\author{
Magdalena Tuczyńska ${ }^{1}$, Maja Matthews-Kozanecka ${ }^{2}$, \\ Arkadiusz Nowak ${ }^{3}$, Ewa Baum ${ }^{2}$ \\ 1 SSC of Maxillofacial Orthopaedics and Orthodontics, Poznan University of Medical \\ Sciences, Poland \\ 2 Department of Social Sciences and the Humanities, Poznan University of Medical Sci- \\ ences, Poland \\ 3 Department of Humanization of Health Care and Sexology, Collegium Medicum, Uni- \\ versity of Zielona Góra, Poland
}

\begin{abstract}
The outbreak of the COVID-19 pandemic had an impact on the global economy, including the provision of health services, with medical facilities and patients cancelling or postponing medical appointments. An alternative to in-person appointments was through the available forms of telemedicine. Scientific reports around the world have suggested that the accessibility and quality of health services declined. The aim of this study was to investigate the accessibility and quality of health services in Poland and to verify whether there were differences between men and women in this respect. The study was based on the authors' own survey questionnaire filled in by 265 respondents, including 181 women, 82 men, and 2 persons without a defined gender. The study revealed that during the COVID-19 pandemic, the accessibility and quality of health services declined. Additionally, women were more likely to use general and specialist health services than men, but a comparison of changes in the assessment of accessibility and quality of services by gender revealed no differences in the assessment of accessibility and quality.
\end{abstract}

\section{Introduction}

Accessibility of health services is a multi-dimensional term that refers to the opportunity to obtain health care when it is wanted or needed. The traditional indicators for measuring the accessibility of health services are the number of doctors or beds per capita. From an economic standpoint, it can be measured in costs to individuals of obtaining health care (Gulliford et al., 2002). Geographic impedance or the number of people who may need the services are also important factors as are patients' opinions, expressed in questionnaires, on the quality of and accessibility of health ser- 
vices (Ma et al., 2018; Quinn et al., 2017). Knowledge of the mechanisms of assessment of patients' satisfaction helps to improve medical facilities. Factors such as waiting time, information reliability, or attention received by patients affect the assessment of the quality of health services. The complexity of measuring patient assessment is related to the moment of measurement, the type of services, or the context (Druică et al., 2019).

On March 11, 2020 the World Health Organization announced a COVID19 pandemic due to the global spread of a novel coronavirus, SARS-CoV2, first identified in Wuhan, China. In most immunocompetent people, the virus causes a mild respiratory infection (Muniyappa \& Gubbi, 2020). The outbreak of COVID-19 affected the economies of most countries around the world, including their health care sectors. Medical facilities were unprepared for the enormous challenges of the huge numbers of patients, the lack of personal protective equipment, and the shortage of medical personnel (Kaye et al., 2020). Some patients had their medical appointments cancelled, others avoided or rescheduled both urgent and regular appointments on their own due to fear of infection (Czeisler et al., 2020). While some appointments required an office visit, others could be made via the telephone (Corazza et al., 2020).

The use of telemedicine during the pandemic was a necessary approach for a continued medical care. It also helped to maintain social distance and reduce the risk of infection for both patients and physicians. For this reason, telemedicine services increased during the pandemic. Unfortunately, telemedicine has its limitations as it requires funding and appropriate guidelines, access to an Internet-enabled device, and measures to secure patients' data. Some people may need help downloading, installing, and using unfamiliar software or applications, which requires additional support. Some physicians may also have a problem with managing cases via telephone. This is why low-income families and citizens of developing countries may be particularly vulnerable to the lack of access to telemedicine (Katzow et al., 2020; Leochico, 2020).

\section{Material and Methods}

The cross-sectional study was conducted among 265 participants, residents Poland, of whom 181 were women, 82 were men, and 2 persons did not define their gender. The inclusion criteria for the study were the completion of a voluntary survey and age above 18 years. Most female respondents were under the age of 60 , most males under the age of 65 . The study was 
conducted using the authors' own survey questionnaire prepared in Polish, i.e. the native language of the respondents. It contained the following questions: 'Did you use medical services during the COVID-19 pandemic, and if so, was it state-funded/self-funded/primary health care?'; 'Did you have any specialist appointments during the COVID-19 pandemic, and if so, what were they?'; 'How do you assess the accessibility to health services before and during the COVID-19 pandemic?', and 'How would you rate the quality of health services before and during the COVID-19 pandemic?'. In the first two questions, the respondents were asked to mark one box. In addition, in the case of an affirmative answer to the first part of the question, the respondents were asked to mark an answer in the second part. In this case, the respondents could mark more than one answer. The last two questions were based on a visual analogue scale (VAS) of 1 to 10, where 1 stood for 'very bad' and 10 for 'very good'. The survey was anonymous, and no personal data that would enable identification of the participants was collected. The questionnaire was distributed in the traditional and online form. No additional consent was taken from the patients to participate in the study.

The calculations were performed using Statistica 13 software from TIBCO. The significance level was $\alpha=0.05$. The result was considered statistically significant when $p<\alpha$. The Mann-Whitney test was performed to compare the variables between 2 groups, while the Kruskal-Wallis test with Dunn's multiple comparisons test was performed for more than 2 groups. To examine changes in the assessment of the accessibility and quality of services, the Wilcoxon test was performed. Spearman's rank correlation coefficient $(R s)$ was performed to compare accessibility and quality and the correlation level was $R s>0$. To examine the relationships between categorical variables, the chi2 test of independence, the Fisher exact test, or the Fisher-Freeman-Halton test were performed.

\section{Results}

Out of the 265 respondents, $211(79.92 \%)$ answered 'yes' to the question of whether they used health services during the COVID-19 pandemic. Among them, 157 (74.41\%) indicated state-funded health services, $120(56.87 \%)$ self-funded, and $70(33.17 \%)$ primary care. A statistically significant result $(p=0.0011)$ was found for gender in relation to the use of medical services; another statistically significant result $(p=0.0019)$ was found for gender in relation to the use of self-funded medical services. The 
other cases, i.e. gender in relation to the use state-funded health services $(p=0.3404)$ and gender in relation to the use of primary care $(p=0.3932)$, were found statistically insignificant.

When asked whether they had any specialist appointments during the COVID-19, 177 (67.04\%) respondents answered 'yes'. Most respondents indicated that they used dental (100, i.e. 56.50\%) and gynaecological care (77, i.e. $43.50 \%)$. Other specialist appointments indicated by the respondents were cardiology (25, i.e. $14.20 \%$ ), general care (27, i.e. $15.34 \%$ ), laryngology (27, i.e. $15.34 \%$ ), psychiatry (10, i.e. 5.68\%), psychology (13, i.e. $7.34 \%$ ), dermatology (34, i.e. $19.21 \%)$, pulmonary care (16, i.e. $9.04 \%)$, neurology (9, i.e. $5.08 \%$ ), orthopaedic care (23, i.e. $12.99 \%)$, urology (11, i.e. $6.21 \%$ ), endocrine care (26, i.e. $14.69 \%)$, oncology (6, i.e. $3.39 \%$ ), ophthalmic care (26, i.e. $14.69 \%)$, nursing care (4, i.e. $2.26 \%$ ), physiotherapy (33, i.e. $18.64 \%)$, and aesthetic medicine (8, i.e. $4.52 \%)$. A statistically significant result $(p=0.0202)$ was also obtained for gender in relation to the use of any specialist medical services, and analysis of the individual specialty showed that only in the case of endocrinology services $(p=0.0009)$ results were statistically significant. Other specialist medical services were found statistically insignificant.

As far as analysing whether the assessment of accessibility and quality of health services was changed by the COVID-19 pandemic is concerned, a statistically significant result was found for both accessibility $(p<0.01)$ and quality $(p<0.01)$ for all participants; in both groups, i.e. female and male, the statistical significance of the results for accessibility $(p<0.01)$ and quality $(p<0.01)$ was the same. The differences are illustrated in Figures 1-6.

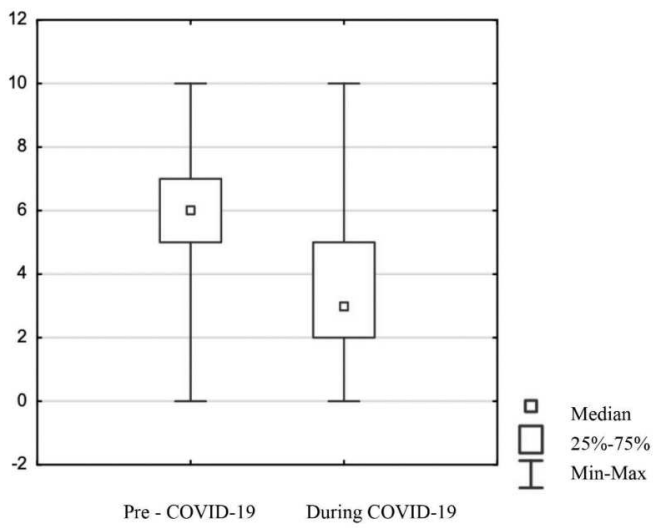

Figure 1. Comparison of scores concerning the access to health services before and during the COVID-19 pandemic for all respondents 
How the COVID-19 Pandemic Affected the Accessibility and Quality...

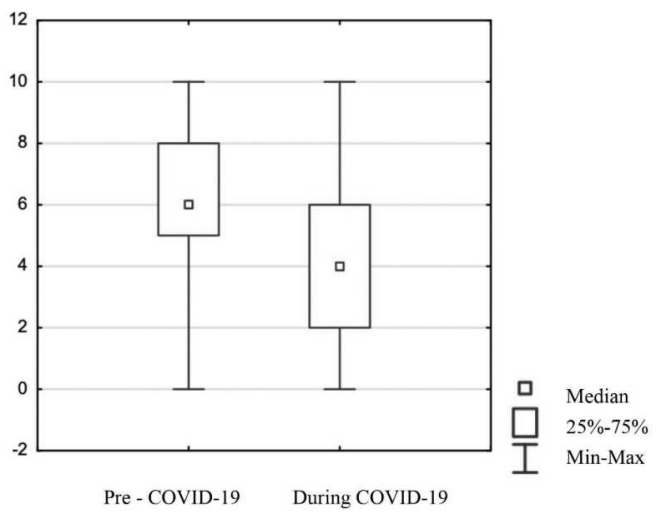

Figure 2. Comparison of scores concerning the quality of health services before and during the COVID-19 pandemic for all respondents

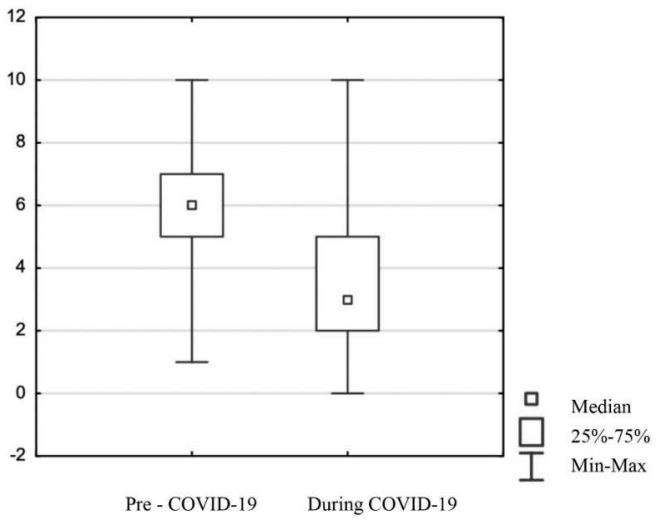

Figure 3. Comparison of scores concerning the access to health services before and during the COVID-19 pandemic among women

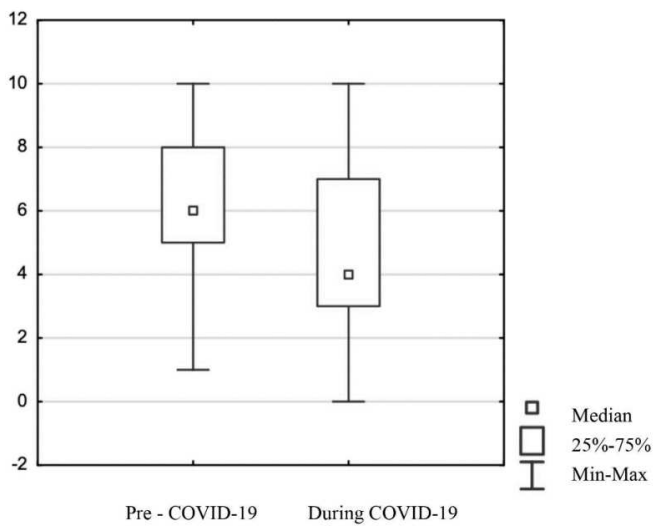

Figure 4. Comparison of scores concerning the quality of health services before and during the COVID-19 pandemic among women 
Magdalena Tuczyńska et al.

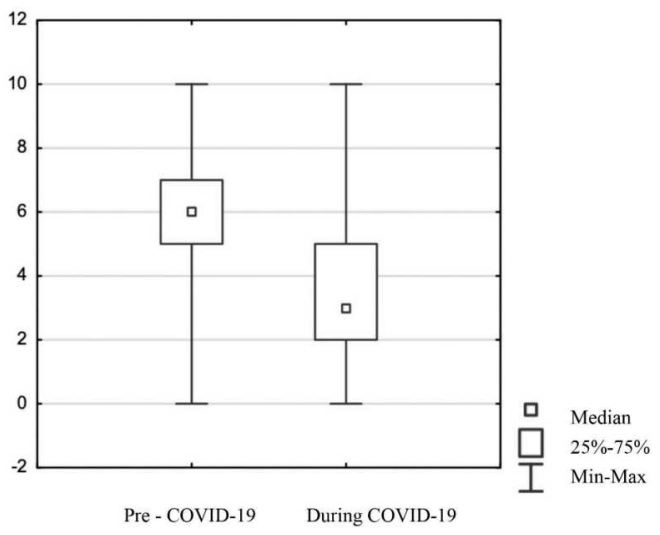

Figure 5. Comparison of scores concerning the access to health services before and during the COVID-19 pandemic among men

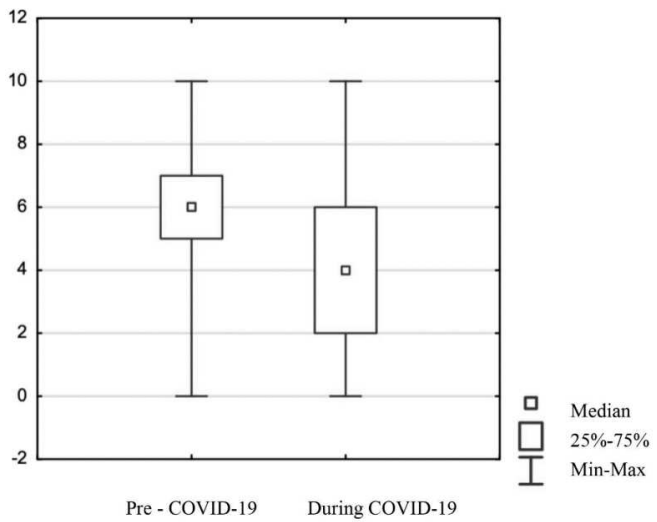

Figure 6. Comparison of scores concerning the quality of health services before and during the COVID-19 pandemic among men

In the Mann-Whitney's test, a comparison of changes in the assessment of accessibility and quality of services by gender revealed no differences, with the following values: for assessment of accessibility $(p=0.4424)$ and for assessment of quality $(p=0.5794)$.

A comparison of whether there existed a correlation between the assessment of accessibility and quality based on Spearman's $R s$ rank correlation showed that the higher the assessment of accessibility of services, the higher the assessment of quality, both before $(R s=0.7703)$ and during $(R s=0.8023)$ the pandemic. The correlation is shown in Figures 7 and 8 . 
How the COVID-19 Pandemic Affected the Accessibility and Quality...

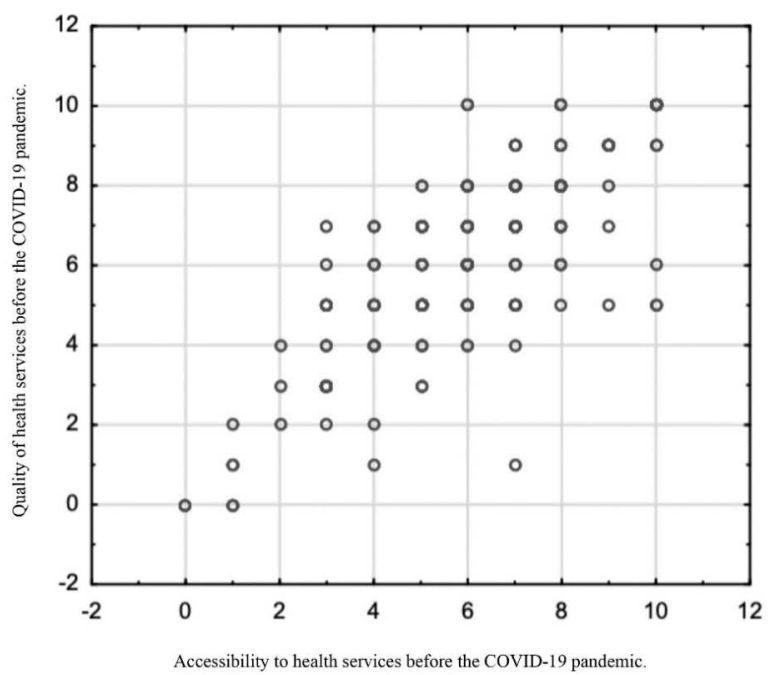

Figure 7. Correlation between accessibility and quality - assessment before the COVID-19 pandemic

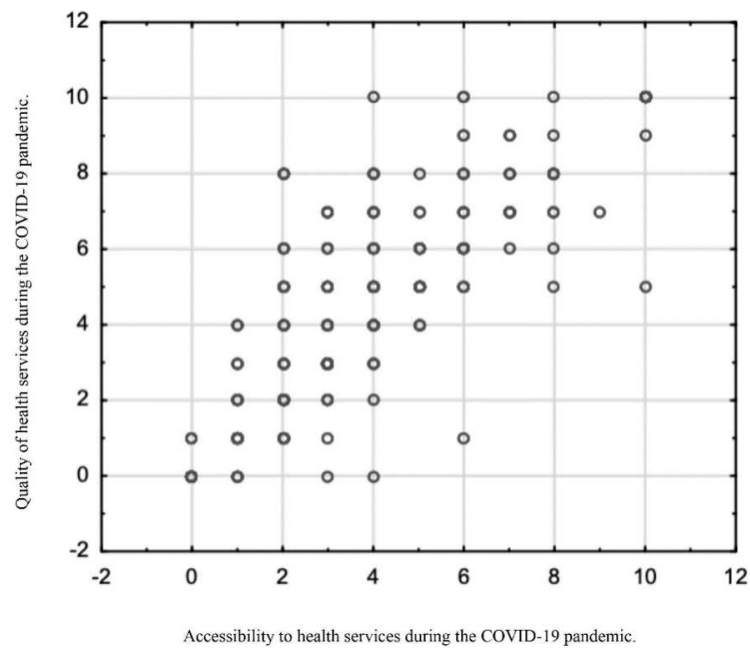

Figure 8. Correlation between accessibility and quality - assessment during the COVID-19 pandemic

Spearman's Rs rank correlation also showed a correlation between change in the assessment of accessibility and change in the assessment of quality. The higher the assessment of accessibility, the higher the assessment of quality $(R s=0.6885)$. Spearman's $R s$ rank correlation is illustrated in Figure 9. 


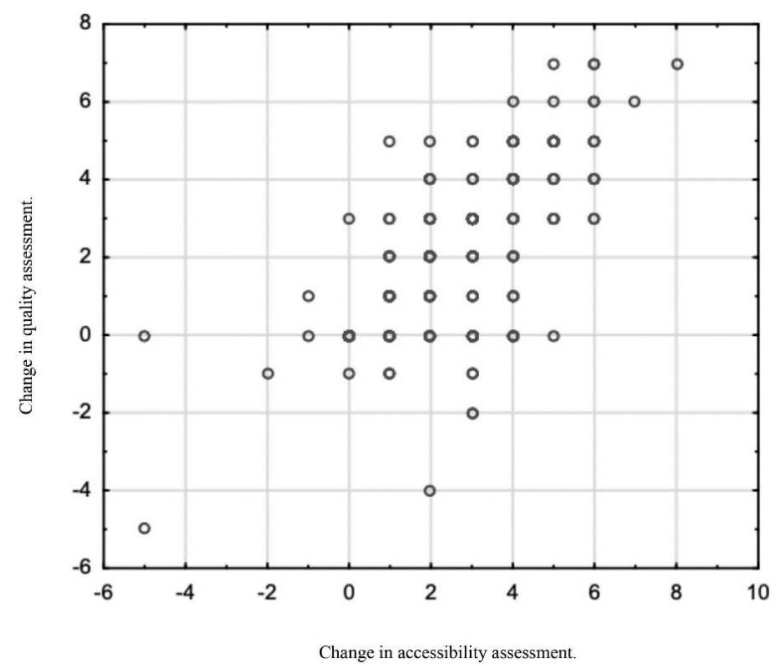

Figure 9. Correlation between change in the assessment of accessibility and change in the assessment of quality

\section{Discussion}

The study has its limitations. In terms of the question concerning the use of general or specialist health services, it does not specify whether the appointment was in-person or via telemedicine. Therefore, this may have resulted in a greater number of affirmative responses, i.e. that respondents used primary care and specialist health services during the pandemic. Despite this, the assessment of accessibility of health services during the pandemic was lower compared to the pre-pandemic period. The quality of health services was also rated lower than before the pandemic. On this basis, it can be concluded that even though their majority of respondents reported using health services during the COVID-19 pandemic, the accessibility and quality declined. Thus, it would be worthwhile to conduct a comprehensive study in the future on a larger study group, one that would include a division into in-person and telemedicine appointments. Another limitation of the study was the lack of data on the accessibility and quality of telemedicine before and during the COVID-19 pandemic. The number of online visits increased during the pandemic, regardless of the age of patients or whether they lived in urban or rural areas (Chu et al., 2021). Expanding the survey to include questions concerning telemedicine services would undoubtedly improve it.

The latest studies conducted worldwide have confirmed concerns about the decline in the accessibility and quality of health services in most med- 
ical fields due to the COVID-19 pandemic outbreak. The reduction was noted in the numbers of visits, hospital admissions, as well as in the area of diagnostics and therapeutics (Moynihan et al., 2021). First-time medical appointments and specialist health care visits also declined during the COVID-19 pandemic period (Nabhen et al., 2020). However, there was an increase in hospital admissions for acute coronary syndromes (ACS) due to the cardiovascular implications of the virus-related systemic inflammatory status. As a result, the mortality rate also increased (D'Ascenzo et al., 2021). Based on the results of an online survey of rheumatic patients in Poland, it was found that $77.57 \%$ of respondents experienced a restriction to health services during the COVID-19 pandemic; $56.06 \%$ of respondents had a specialist clinic appointment cancelled; $31.77 \%$ stated that they did not receive medical care when needed, while $16.04 \%$ noted an improvement in the quality of telemedicine services (Opinc et al., 2021).

Before the outbreak of the COVID-19 pandemic, many survey databased studies presented results which showed that women were more likely to show up for medical appointments than men (Wang et al., 2013). This study revealed that during the pandemic, women were indeed more likely to use general and specialist health services. Regarding general health services, women were more likely to use the private sector services than men. Moreover, there was no difference in gender concerning the use of state-funded and primary care services. In addition, with respect to specialist health services, women were more likely to use endocrinology services. There were no differences in the assessment of the accessibility and quality between women and men.

One of the several indicators of the quality of medical services is patients' satisfaction; during the pandemic, its level was deficient. Access to medication, disinfectants, personal protective equipment, and maintaining social distance impacted this assessment (Deriba et al., 2020). Regardless of the state of the particular country's development, almost all people had to face the challenges associated with the SARS-CoV2 virus related to preventing disease transmission, diagnosing new cases, providing care, and ensuring recovery (Rodela et al., 2020). Access to health care in developing countries had already been limited before the pandemic, and even where health care is available, the quality is often severely deficient. Many people suffer from diseases such as diarrhoea, pneumonia, hypertension, or malaria, for which at least one effective treatment exists. The pandemic made access to medicines even more restricted due to a noticeable decrease in production and exportation across countries. Nwoke Emmanuel et al. (2020) conducted a questionnaire-based study among Nigerian people, which showed that re- 
spondents with a chronic disease had more difficulty accessing medications during the pandemic than before, significantly exacerbating their health condition. In addition, an increase in the costs of medicines was also noted (O’Donnell, 2007).

The Declaration of Human Rights assumes that everyone should receive optimal health care. Society's concerns were related to the deprivation of the right to primary care, and the reduction in the number of patients, treatments, and therapies introduced by hospitals and clinics due to the SARS-CoV2 virus. Various moral dilemmas arose during the COVID-19 pandemic, and although many of these could have been resolved with the use of the Code of Medical Ethics, a critical time such as a pandemic requires extraordinary ethical standards (Baum \& Żok, 2020). The increase in the demand for ICU beds modified the criteria for patient selection, i.e. in case of shortage of ICU beds, they were more frequently considered as necessitating mainly palliative comfort care. The idea of creating several units with ICU beds outside the hospital proved to be a good solution regarding transfers of ICU patients to distant locations where ICU beds are available (Robert et al., 2020).

\section{Conclusions}

One of the fundamental human rights is the right of access to health services without suffering financial hardship. The COVID-19 pandemic has undoubtedly been an economic and ethical challenge for all countries worldwide and their health care systems. The lack of personal protective equipment and medical personnel, the fear of infection, and the high numbers of patients - all of these contributed to a decrease in the quality and accessibility of health services. In Poland, patients use state and self-funded health services, but the quality and accessibility of those services declined, an opinion expressed regardless of gender.

\section{R E F E R E N C E S}

Baum, E., \& Żok, A. (2020). Assumptions of maximal individualism in the time of the COVID-19 pandemic: ethical aspects. Society Register, 4(3), 79-88. doi: https://doi.org/10.14746/sr.2020.4.3.04

Bokolo Anthony Jnr (2020). Use of Telemedicine and Virtual Care for Remote Treatment in Response to COVID-19 Pandemic. Journal of medical systems, 44(7), 132. doi: https://doi.org/10.1007/s10916-020-01596-5 
How the COVID-19 Pandemic Affected the Accessibility and Quality...

Corazza, P., D'Alterio, F. M., \& Younis, S. (2020). Proposed algorithm during COVID-19 pandemic for patient management in medical retina clinic. International journal of retina and vitreous, 6, 20. doi: https://doi.org/10.1186/ s40942-020-00226-z

Chu, C., Cram, P., Pang, A., Stamenova, V., Tadrous, M., \& Bhatia, R. S. (2021). Rural Telemedicine Use Before and During the COVID-19 Pandemic: Repeated Cross-sectional Study. Journal of medical Internet research, 23(4), e26960. doi: https://doi.org/10.2196/26960

Czeisler, M. É., Marynak, K., Clarke, K., Salah, Z., Shakya, I., Thierry, J. M., Ali, N., McMillan, H., et al. (2020). Delay or Avoidance of Medical Care Because of COVID-19-Related Concerns - United States, June 2020. MMWR. Morbidity and mortality weekly report, 69(36), 1250-1257. doi: https://doi. org/10.15585/mmwr.mm6936a4

D’Ascenzo, F., De Filippo, O., Borin, A., Barbieri, L., Adamo, M., Morici, N., Truffa Giachet, A., et al. (2021). Impact of COVID-19 pandemic and infection on in hospital survival for patients presenting with acute coronary syndromes: A multicenter registry. International journal of cardiology, 332, 227-234. doi: https://doi.org/10.1016/j.ijcard.2021.03.063

Deriba, B. S., Geleta, T. A., Beyane, R. S., Mohammed, A., Tesema, M., \& Jemal, K. (2020). Patient Satisfaction and Associated Factors During COVID19 Pandemic in North Shoa Health Care Facilities. Patient preference and adherence, 14, 1923-1934. doi: https://doi.org/10.2147/PPA.S276254

Druică, E., Mihăilă, V., Burcea, M., \& Cepoi, V. (2019). Combining Direct and Indirect Measurements to Assess Patients' Satisfaction with the Quality of Public Health Services in Romania: Uncovering Structural Mechanisms and Their Implications. International journal of environmental research and public health, 17(1), 152. doi: https://doi.org/10.3390/ijerph17010152

Gulliford, M., Figueroa-Munoz, J., Morgan, M., Hughes, D., Gibson, B., Beech, R., \& Hudson, M. (2002). What does 'access to health care' mean?. Journal of health services research \& policy, 7(3), 186-188. doi: https://doi.org/10. $1258 / 135581902760082517$

Katzow, M. W., Steinway, C., \& Jan, S. (2020). Telemedicine and Health Disparities During COVID-19. Pediatrics, 146(2), e20201586. doi: https://doi.org/10. 1542/peds.2020-1586

Kaye, A. D., Okeagu, C. N., Pham, A. D., Silva, R. A., Hurley, J. J., Arron, B. L., Sarfraz, N., Lee, H. N. et al. (2020). Economic impact of COVID-19 pandemic on healthcare facilities and systems: International perspectives. Best Practice \& Research. Clinical Anaesthesiology, Advance online publication. doi: https://doi.org/10.1016/j.bpa.2020.11.009

Leochico, C. F. D. (2020). Adoption of telerehabilitation in a developing country before and during the COVID-19 pandemic. Annals of physical and rehabilitation medicine, 63(6), 563-564. doi: https://doi.org/10.1016/j.rehab.2020. 06.001 
Ma, L., Luo, N., Wan, T., Hu, C., \& Peng, M. (2018). An Improved Healthcare Accessibility Measure Considering the Temporal Dimension and Population Demand of Different Ages. International journal of environmental research and public health, 15(11), 2421. doi: https://doi.org/10.3390/ijerph15112421

Moynihan, R., Sanders, S., Michaleff, Z. A., Scott, A. M., Clark, J., To, E. J., Jones, M., Kitchener, E. et al. (2021). Impact of COVID-19 pandemic on utilisation of healthcare services: a systematic review. BMJ open, 11(3), e045343.

Muniyappa, R., \& Gubbi, S. (2020). COVID-19 pandemic, coronaviruses, and diabetes mellitus. American journal of physiology. Endocrinology and metabolism, 318(5), E736-E741. doi: https://doi.org/10.1152/ajpendo.00124.2020

Nabhen, J. J., Ostroski, T., Kozonoe, M. M., Orlandi, D., Tormen, T., \& Ioshii, S. O. (2020). Impact of the COVID-19 pandemic in patient admission to a highcomplexity cancer center in Southern Brasil. Revista da Associacao Medica Brasileira (1992), 66(10), 1361-1365. doi: https://doi.org/10.1590/18069282.66.10.1361

Nwoke, E. A., Ofomata, C. J., Amadi, C. M., Jibuaku, C. H., Akahome, I. D., \& Nwagbo, E. C. (2020). Impact of the COVID-19 Pandemic on Consumers' Access to Essential Medicines in Nigeria. The American journal of tropical medicine and hygiene, 103(4), 1630-1634. doi: https://doi.org/10.4269/ajtmh. 20-0838

O'Donnell O. (2007). Access to health care in developing countries: breaking down demand side barriers. Cadernos de saude publica, 23(12), 2820-2834. doi: https://doi.org/10.1590/s0102-311x2007001200003

Opinc, A., Brzezińska, O., \& Makowska, J. (2021). The impact of COVID-19 and healthcare system changes on the well-being of rheumatic patients. Reumatologia, 59(2), 73-84. doi: https://doi.org/10.5114/reum.2021.105424

Quinn, M., Robinson, C., Forman, J., Krein, S. L., \& Rosland, A. M. (2017). Survey Instruments to Assess Patient Experiences With Access and Coordination Across Health Care Settings: Available and Needed Measures. Medical care, 55 Suppl 7 Suppl 1 (Suppl 7 1), S84-S91. doi: https://doi.org/10.1097/MLR. 0000000000000730

Robert, R., Kentish-Barnes, N., Boyer, A., Laurent, A., Azoulay, E., \& Reignier, J. (2020). Ethical dilemmas due to the Covid-19 pandemic. Annals of intensive care, 10(1), 84. doi: https://doi.org/10.1186/s13613-020-00702-7

Rodela, T. T., Tasnim, S., Mazumder, H., Faizah, F., Sultana, A., \& Hossain, M. (2020, April 4). Economic Impacts of Coronavirus Disease (COVID-19) in Developing Countries. doi: https://doi.org/10.31235/osf.io/wygpk

Wang, Y., Hunt, K., Nazareth, I., Freemantle, N., Petersen, I. (2013). Do men consult less than women? An analysis of routinely collected UK general practice data. BMJ Open, 19, 3(8), e003320. doi: 10.1136/bmjopen-2013-003320 